\title{
Unsupervised Active Regions for Multiresolution Image Segmentation
}

\author{
X.Muñoz, J.Martí, X.Cufí and J.Freixenet \\ University of Girona \\ Institute of Informatics and Applications. \\ Campus de Montilivi s/n. 17071. Girona, Spain \\ \{xmunoz,joanm,xcuf,jordif\}@eia.udg.es
}

\begin{abstract}
An unsupervised approach to image segmentation which fuses region and boundary information is presented. The proposed approach takes advantage of the combined use of 3 different strategies: the guidance of seed placement, the control of decision criterion, and the boundary refinement. The new algorithm uses the boundary information to initialize a set of active regions which compete for the pixels in order to segment the whole image. The method is implemented on a multiresolution representation which ensures noise robustness as well as computation efficiency. The accuracy of the segmentation results has been proven through an objective comparative evaluation of the method.
\end{abstract}

\section{Introduction}

Image segmentation is one of the most important processes of image analysis, understanding and interpretation. Many segmentation methods are based on two basic properties of the pixels in relation to their local neighbourhood: discontinuity and similarity. Methods based on some discontinuity property of the pixels are called boundary-based methods, whereas methods based on some similarity property are called region-based methods. Unfortunately, both techniques, boundary-based and region-based, often fail to produce accurate segmentation results [8]

With the aim of improving the segmentation process, a large number of new algorithms which integrate region and boundary information have been proposed over the past few years. Taking into account the complementary nature of the boundary-based and region-based information, it is possible to alleviate the problems related to each when considered separately. Although it is assumed that integration of both methods yields to get complementary information and, therefore, has long been a highly desirable goal [8], it becomes a non-trivial task due to the conflicts and incommensurate objectives it involves.
The existing main strategies which integrate region and boundary information [2] rely on the processes of guidance of seed placement (where boundary information is used in order to decide the most suitable position to place the seed of the region growing process $[3,6]$ ), control of decision criterion (the inclusion of edge information in the definition of the decision criterion which controls the growth of the region $[4,10]$ ), and boundary refinement (where boundary information is used to refine the inaccurate boundaries obtained by a region based segmentation $[1,9])$.

In this paper we propose a new segmentation method which combines these strategies: taking as a basis a previously developed segmentation algorithm [3], we have used the main contours of the image to adequately place the seeds in order to initialize the region models; then as these regions grow, they compete for the pixels of the image by using a decision criterion which ensures the homogeneity inside the region and the presence of edges at its boundary; finally, the method has been implemented on a multiresolution representation which allows us to refine the region boundaries from a coarse to a finer resolution.

The remainder of this paper is structured as follows: Section 2 describes the proposed region segmentation technique, detailing the placement of the starting seeds and the growing of the active regions. The multiresolution representation is presented in Section 3, while experimental results proving the validity of our proposal appear in Section 4 . Finally, conclusions are given in Section 5.

\section{Active Region Segmentation}

Recently, the concept of active regions as a way to combine the region and boundary information has been introduced. This model is a considerable extension on the active contour model since it incorporates region-based information with the aim of finding a partition where the interior and the exterior of the region preserve the desired image properties. The underlying idea is that the region moves through 
the image (shrinking or expanding) in order to contain a single, whole region. The competition algorithm proposed by $\mathrm{Zhu}$ and Yuille [12] and the geodesic active regions presented in Paragios and Deriche's work [7] are good examples of active region models.

The main contribution of our proposal is twofold:

- Unsupervised region initialization: the seeds which allow us to initialize the statistical measurements which model the region are automatically placed from the boundary information. Hence, it is not necessary user intervention or a previous learning phase.

- Integrated energy function: the energy function incorporates the homogeneity inside the region (region information) and the discontinuity at the contour (boundary information).

\subsection{Initialization}

To obtain a sample of each region large enough to model its homogeneity behaviour, initial seeds have to be placed completely inside the regions. Boundary information allows us to extract these positions in the "core" of the regions by looking for places far away from the contours. The seed placement is realised according to the algorithm proposed in [3]. The method is based on the detection and extraction of the most relevant contours in the image characterized by an outstanding length within the global frame of the image and by any appreciable difference between the separated regions in chromatic and textural features. Further, seeds are placed in zones free of contours or, in other words, the "core" of the regions.

Each region is modeled by a Gaussian distribution, so the mean and the standard deviation, which are initialized from the seeds, describe the homogeneity region behaviour. Hence, the probability of a pixel $(x, y)$ of belonging to a region characterized by $(\mu, \sigma)$ is

$$
P_{R}((x, y) \mid(\mu, \sigma))=\frac{1}{\sqrt{2 \pi} \sigma} \exp \left\{-\frac{\left(I_{(x, y)}-\mu\right)^{2}}{2 \sigma^{2}}\right\}
$$

where $I_{(x, y)}$ is the intensity of the pixel $(x, y)$. The background is treated as a single region having uniform probability distribution $P_{0}$.

\subsection{Active Region Growing}

The goal of image segmentation is to partition the image into subregions with homogeneous intensity (color or texture) properties in its interior and a high discontinuity with neighbouring regions in its boundary. With the aim of integrating both conditions in an optimal segmentation, the global energy is defined with two basic terms.
Boundary term measures the probability that boundary pixels are really edge pixels. The probability of a given pixel $(x, y)$ being at the real boundary is measured by $P_{B}((x, y))$, which can be considered as directly proportional to the value of the magnitude gradient of the pixel. Meanwhile, region term measures the homogeneity in the interior of the regions by the probability that these pixels belong to each corresponding region. As has been previously defined, $P_{R}((x, y) \mid(\mu, \sigma))$ measures the probability that a pixel $(x, y)$ belongs to a region modeled by $(\mu, \sigma)$.

Some complementary definitions are required: let $\rho(R)=\left\{R_{i}: i \epsilon[0, N]\right\}$ be a partition of the image into $\{N+1\}$ non-overlapping regions, where $R_{0}$ is the region corresponding to the background region. Let $\partial \rho(R)=$ $\left\{\partial R_{i}: i \epsilon[1, N]\right\}$ be the region boundaries of the partition $\rho(R)$. The energy function is defined as

$$
\begin{aligned}
& E(\rho(R))= \\
& \left.(1-\alpha) \sum_{i=1}^{N}-\log P_{B}\left((x, y):(x, y) \epsilon \partial R_{i}\right)\right) \\
& +\alpha \sum_{i=0}^{N}-\log P_{H}\left((x, y):(x, y) \epsilon R_{i} \mid\left(\mu_{i}, \sigma_{i}\right)\right)
\end{aligned}
$$

where $\alpha$ is a model parameter weighing the two terms: boundary probability and region homogeneity. This function is then optimized by a greedy algorithm which takes into account the neighbouring pixels to the current region boundaries $\partial \rho(R)$ to determine the next movement. Concretely, a region aggregates a neighbouring pixels when this new classification diminishes the energy of the segmentation.

Intuitively, all regions begin to move and grow, competing for the pixels of the image until an energy minimum is reached. When the minimization process finishes, if there is a background region $R_{0}$ not occupied by any seed regions, a new seed is placed in the background, and the energy minimization starts again.

\section{MultiResolution Implementation}

In order to further reduce the computational cost, a multiscale representation [9] is proposed which can be combined with the active region segmentation. Specifically, a pyramid of images at different scales is built upon the full resolution image. At lowest resolution level, the seeds are placed from the boundary information and start to compete for the image, obtaining a first segmentation result. This multiresolution structure is then used according to a coarse-to-fine strategy which assumes the invariance of region properties over a range of scales. Specifically, a boundary region is defined at coarsest level and then, at successive levels, the pixels not classified as boundary, the core of the region, are used to initialize and model the regions. A scheme is depicted in Figure 1. Further, segmentation by 


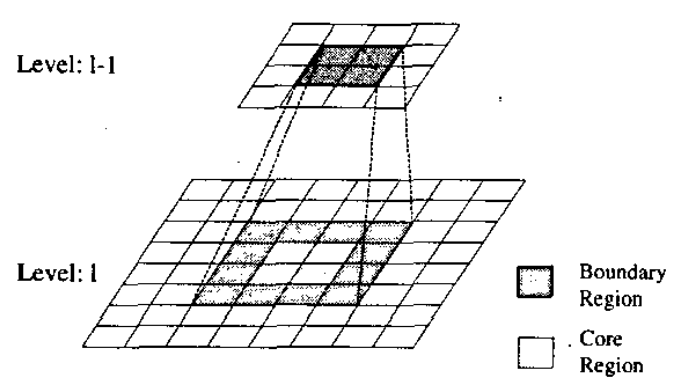

Figure 1. Pyramid of image resolutions. The segmentation results obtained at level I-1 are propagated to level I, where not boundary pixels are used to model the region.

active region is performed to refine the candidate boundary by a factor of two using the multiresolution structure. As a result, the boundaries of the full image size are produced at the finest resolution.

Furthermore, the use of a multiresolution representation allows us to avoid the over-segmentation problems produced by the presence of noise in images. An initial coarse region segmentation is performed on a lower resolution achieving the effect of smoothing. Hence, the use of a multiresolution technique ensures noise robustness as well as computation efficiency.

\section{Experimental Results}

The performance of our proposal has been analyzed over a set of 22 test images including synthetic and real ones. The set of 12 synthetic images has been generated following the method proposed by Zhang [11], where the form of the objects of the images changes from a circle to an elongated ellipse. To make synthetic images more realistic, a $5 x 5$ average low-pass filter is applied to produce a smooth transition between objects and background. Then, a zeromean Gaussian white noise is added to simulate noise effect. On the other hand, 10 selected real images are well-known standard test images extracted from the USC-SIPI image database (University of Southern California-Signal and Image Processing Institute). All test images are size $256 \times 256$ pixels. Figure 2 shows an example of segmentation with three images belonging to the trial set.

In order to evaluate the results, we use the quality parameters (region-based and boundary-based) proposed by Huang and Dom [5]. The boundary-based approach evaluates segmentation in terms of both localization and shape
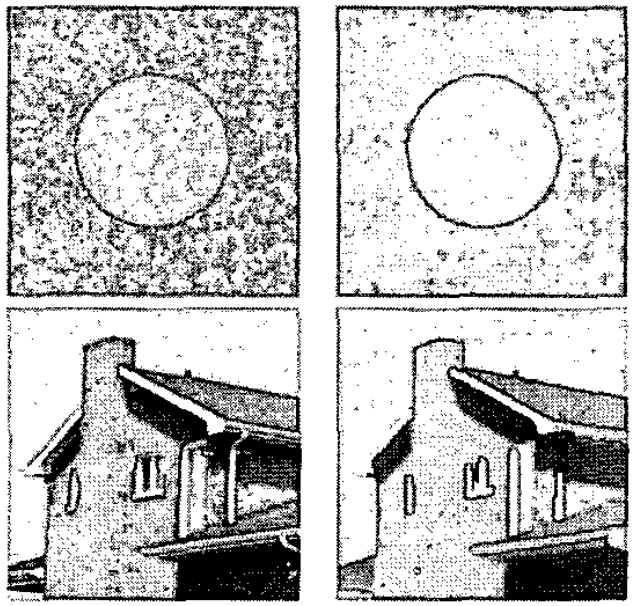

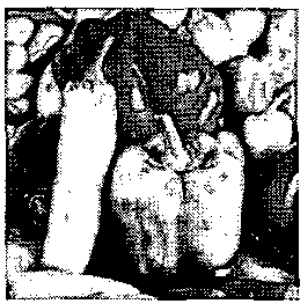

(a)

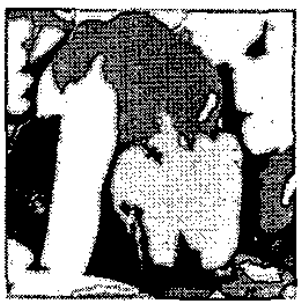

(b)
Figure 2. The segmentation results obtained over three images of the trial set. (a) Original images, (b) segmentation results.

accuracy of extracted regions. Two distance distribution signatures are used to measure the boundary quality, one from ground truth to the estimated, denoted by $D_{G}^{B}$, and the other from the estimated to ground truth, denoted by $D_{B}^{G}$. Instead, the region-based approach assesses the segmentation quality in terms of both size and location of the segmented regions. A region-based performance measure based on normalized Hamming distance is defined, $p$. Moreover, two types of errors are defined: missing rate $e_{k}^{m}$ and false alarm rate $e_{R}^{f}$.

Furthermore, we have made a comparison of our proposal against other strategies to integrate region and boundary information. Thereby, we implemented the algorithms corresponding to the three previously described strategies. Concretely, our implementation of the guidance of the seed placement strategy (GSP) is based on proposal [3], while the control of decision criterion strategy (CDC) is based on [10], and the boundary ref inement strategy (BR) on [9]. Table 1 shows the summarized results obtained for the three strategies; 
Table 1. Summarized segmentation results of main strategies and our proposal over a set of 12 synthetic and 10 real test images.

\begin{tabular}{|c|c|c|c|c|c|c|c|c|}
\hline \multirow[t]{2}{*}{ Strategy } & \multicolumn{3}{|c|}{ Region-based } & \multicolumn{4}{|c|}{ Boundary-based } & \multirow[t]{2}{*}{ Time } \\
\hline & $e_{H}^{m}$ & $e_{t}^{J}$ & $\boldsymbol{p}$ & $\mu D_{G}^{B}$ & $\sigma D_{G}^{B}$ & $\mu D_{B}^{G}$ & $\sigma D_{B}^{G}$ & \\
\hline \multicolumn{9}{|c|}{ Summary of Synshetic Images Evaluation } \\
\hline GSP & 0.059 & 0,025 & $0, \overline{957}$ & 1,003 & 0,708 & 0.993 & 0.843 & 0,340 \\
\hline $\mathrm{CDC}$ & 0.202 & 0,011 & 0,893 & 0,954 & 0,658 & 0,965 & 0,877 & 0.180 \\
\hline BR & 0,034 & 0,066 & 0,9 & 0,404 & 0,506 & 0,765 & 0,771 & 0,060 \\
\hline $\mathbf{A R}$ & 0,089 & 0,015 & 0.947 & 0,480 & 0.312 & 0,563 & 0,688 & 6,251 \\
\hline MARS & 0,030 & 0,015 & 0,977 & 0,475 & 0.580 & 0,560 & 0.341 & 0,980 \\
\hline \multicolumn{9}{|c|}{ Summan of Real Images Evaluation } \\
\hline GSP & 0,066 & 0,033 & 0,949 & 2,121 & 3,018 & 2,160 & 3,389 & 0,430 \\
\hline $\mathrm{CDC}$ & 0,174 & $0,04 \overline{4}$ & 0.890 & 1,828 & 1.297 & 1,544 & 3.125 & 0,360 \\
\hline BR & 0,074 & 0,088 & 0,918 & 0,698 & 0,968 & 0.412 & 0.982 & 0,076 \\
\hline ARS & 0,106 & 0.033 & 0,929 & 0.711 & 0.528 & 0.344 & 0,886 & 8,132 \\
\hline MARS & 0,038 & 0,032 & 0,964 & 0.730 & 0.711 & 0,320 & 0,641 & 1,256 \\
\hline
\end{tabular}

our proposal of active region segmentation (ARS), and our proposal implemented on a multiresolution representation (MARS).

Quantitative results successfully demonstrate the validity of our proposal. Although the active region segmentation (ARS) obtains useful results, the technique has some problems due to the presence of noise which causes an oversegmentation of the image and a relatively high computational cost. The implementation of this proposal on a multiresolution representation (MARS) solves these problems and achieves an optimal result.

\section{Conclusions}

A new strategy for image segmentation which integrates region and boundary information has been described. The algorithm uses boundary information in order to initialize, in an unsupervised way, a set of active regions, which later compete for the pixels minimizing an energy function which takes into account both region and boundary information. The method has been implemented on a multiresolution representation and has been tested on a set of synthetic and real images. The experimental results demonstrate the effectiveness of the proposed algorithm in estimating regions and their boundaries with high accuracy.

The algorithm can be directly adapted to perform color or texture segmentation assuming multi-variable Gaussian distributions to model each region. In this sense, future extensions of this work are oriented to the integration of grey level, color and texture cues.

\section{Acknowledgments}

This study has been partially developed thanks to the support of the Departament d'Universitats, Recerca $i$ Societat de la Informació de la Generalitat de Catalunya.

\section{References}

[1] F. Chan, F. Lam, P. Poon, H. Zhu, and K. Chan. Object boundary location by region and contour deformation. IEE Proceedings-Vision Image and Signal Processing. 143(6):353-360, December 1996.

[2] X. Cufí, X. Muñoz, J. Freixenet, and J. Marti. A review on image segmentation techniques integrating region and boundary information. Advances in Imaging and Electronics Physics, 2002.

[3] X. Cufí, X. Muñoz, J. Freixenet, and J. Marti. A concurrent region growing algorithm guided by circumscribed contours. In International Conference on Pattern Recognition, volume I, pages 432-435, Barcelona. Spain. September 2000.

[4] R. Falah, P. Bolon, and J. Cocquerez. A region-region and region-edge cooperative approach of image segmentation. In International Conference on Image Processing, volume 3, pages 470-474, Austin, Texas, October 1994.

[5] Q. Huang and B. Dom. Quantitative methods of evaluating image segmentation. In International Conference on Image Processing, volume III, pages 53-56, Washington DC, October 1995.

[6] A. Moghaddamzadeh and N. Bourbakis. A fuzzy region growing approach for segmentation of color images. Pattern Recognition, 30(6):867-881, 1997.

[7] N. Paragios and R. Deriche. Geodesic active regions for supervised texture segmentation. In International Conference on Computer Vision, volume II, pages 926-932, Corfou, Greece, September 1999.

[8] T. Pavlidis and Y. Liow. Integrating region growing and edge detection. IEEE Transactions on Pattern Analysis and Machine Intelligence, 12(3):225-233, March 1990

[9] R. Wilson and M. Spann. Finite prolate spheroidial sequences and their applications ii: Image feature description and segmentation. IEEE Transactions on Pattern Analysis and Machine Intelligence, 10(2):193-203, 1988.

[10] Y. Xiaohan, J. Yla-Jaaski, O. Huttunen, T. Vehkomaki, $\mathrm{O}$. Sipild, and T. Katila. Image segmentation combining region growing and edge detection. In International Conference on Pattern Recognition, volume C, pages 481-484, The Hague, Netherlands, August 1992.

[11] Y. Zhang. Evaluation and comparison of different segmentation algorithms. Pattern Recognition Letters, 18:963-974, 1997.

[12] S. Zhu and A. Yuille. Region competition: Unifying snakes, region growing, and bayes/mdl for multi-band image seg. mentation. IEEE Transactions on Pattern Analysis and Machine Intelligence, 18(9):884-900, September 1996. 